\title{
Jak1/Stat3 Activation Alters Phosphate Metabolism Independently of Sex and Extracellular Phosphate Levels
}

\author{
Nicole Gehring $^{\text {a Carla Bettoni }}{ }^{\mathrm{a}}$ Carsten A. Wagner ${ }^{\mathrm{a}}$ Isabel Rubio-Aliaga ${ }^{\mathrm{a}}$ \\ anstitute of Physiology, University of Zurich, National Center of Competence in Research NCCR Kidney.CH, Zurich, \\ Switzerland
}

\section{Keywords}

Jak1/Stat3 signaling · FGF23 · Sex differences · Phosphate

\begin{abstract}
Introduction: Phosphate homeostasis is regulated by a complex network involving the parathyroid hormone (PTH), fibroblast growth factor 23 (FGF23), and calcitriol acting on several organs including the kidney, intestine, bone, and parathyroid gland. Previously, we showed that activation of the Janus kinase 1 (Jak1)-signal transducer and activator of transcription 3 (Stat3) signaling pathway leads to altered mineral metabolism with higher FGF23 levels, lower PTH, and higher calcitriol levels. Here, we investigated if there are sex differences in the role of Jak1/Stat3 signaling pathway on phosphate metabolism and if this pathway is sensitive to extracellular phosphate alterations. Methods: We used a mouse model (Jak $1^{\mathrm{S645 \textrm {P } + / -})}$ that resembles a constitutive activating mutation of the Jak1/Stat3 signaling pathway in humans and analyzed the impact of sex on mineral metabolism parameters. Furthermore, we challenged Jak $1^{\mathrm{S} 645 \mathrm{P}+/-}$ male and female mice with a high $(1.2 \% \mathrm{w} / \mathrm{w})$ and low $(0.1 \% \mathrm{w} / \mathrm{w})$ phosphate diet and a diet with phosphate with organic origin with lower bioavailability. Results: Female mice, as male mice, showed higher intact FGF23 levels but no phosphaturia, and higher calcitriol and lower PTH levels in plasma. A
\end{abstract}

phosphate challenge did not alter the effect of Jak1/Stat3 activation on phosphate metabolism for both genders. However, under a low phosphate diet or a diet with lower phosphate availability, the animals showed a tendency to develop hypophosphatemia. Moreover, male and female mice showed similar phosphate metabolism parameters. The only exception was higher PTH levels in male mice than those in females. Discussion/Conclusion: Sex and extracellular phosphate levels do not affect the impact of Jak1/Stat3 activation on phosphate metabolism.

(C) 2021 The Author(s)

Published by S. Karger AG, Basel

\section{Introduction}

The kidney plays a predominant role in the regulation of phosphate metabolism [1-4]. Fibroblast growth factor 23 (FGF23), parathyroid hormone (PTH), and 1,25(OH) vitamin $\mathrm{D}_{3}$ (calcitriol) are the key hormones in the regulation of phosphate (and calcium) metabolism. The reciprocal regulation of these hormones forms a complex network, acting mainly on the kidney [1-4]. Briefly, both FGF23 and PTH regulate calcitriol synthesis and inactivation in the kidney. Calcitriol induces FGF23 synthesis in the bone. PTH promotes calcitriol and FGF23 synthesis and stimulates phosphate urinary excretion. FGF23 is

Correspondence to:

Carsten A. Wagner, carsten.wagner@physiol.uzh.ch

C 2021 The Author(s)

Published by S. Karger AG, Basel

www.karger.com/kbr

This article is licensed under the Creative Commons Attribution 4.0 
a phosphaturic hormone, which also inhibits calcitriol synthesis and induces its inactivation in the kidney. Furthermore, FGF23 reduces PTH synthesis in the parathyroid gland. Alterations of phosphate homeostasis are found in a variety of disorders including chronic kidney disease. Moreover, both hyper- and hypophosphatemia are detrimental for health, affecting mainly renal and cardiovascular function and bone health [5]. Understanding further regulators of this complex, network is of major importance.

Recently, we have shown that the Janus kinase (JAK)signal transducer and activator of transcription (STAT) pathway alters the metabolism of the hormones regulating phosphate homeostasis $[6,7]$. The JAK/STAT pathway is an evolutionarily conserved signaling pathway that mediates the action of cytokines, hormones, and growth factors to control cell growth and differentiation [8]. Increased activation of JAK/STAT signaling leads to disease, mainly cancer and many immune-mediated inflammatory diseases. The Jak family comprises 4 members: JAK1, JAK2, and TYK2 with a wide distribution in the organism and JAK3, which is predominantly expressed in hematopoietic cells. Ablation of Jak3 function in mice leads to high calcitriol and FGF23 levels in the plasma [9]. We showed that constitutive and systemic activation of Jak1 highly increases active and cleaved FGF23 levels, lowers PTH, and increases calcitriol levels in the plasma [6]. Activation of the Jak1/Stat3 pathway highly stimulated FGFG23 production; however, most circulating FGF23 was cleaved to cause excessive levels of the C-terminal FGF23 fragment. This C-terminal fragment has been shown to inhibit the function of intact FGF23 [10], and we showed that the plasma from mice with the Jak1 mutation inhibited intact FGFG23 signaling. Moreover, this constitutive Jak 1 activation led to stunted growth and altered bone physiology [7]. These findings were described investigating mainly male mice. In the last decade, investigating both sexes in science has become a major interest, as it has been for long recognized that both sex and gender are modifiers of health and disease outcomes [11]. Moreover, diagnosis, drug action, and therapies are also affected by gender and sex. Chronic kidney disease is more prevalent in women than men and is also among the ten leading causes of death for women but not for men $[12,13]$. Women with chronic kidney disease have a higher risk of developing cardiovascular disease than men [14]. Phosphate metabolism shows sex differences as observed in human studies and rodent experiments. Plasma phosphate levels are highest after birth and fall continuously until the end of puberty [15]. Thereafter, plasma levels remain constant in men, whereas they significantly increase in women and remain higher, which is also reflected in a higher tubular threshold for phosphate (TmP/GFR) in postmenopausal women [16]. In mice, estrogen downregulates the expression of renal phosphate transporters NaPi-IIa and NaPi-IIc [17], while it stimulates active intestinal phosphate transport [18]. Therefore, in order to further understand the effect of sex on phosphate metabolism, here, we compared how phosphate metabolism is altered in male and female mice following Jak1/Stat3 activation.

The mechanisms behind mammalian phosphate sensing are not fully understood $[19,20]$. Extracellular phosphate is a signaling molecule modulating the expression of several genes in bone cells, such as osteopontin, dentin matrix acidic phosphoprotein 1, cyclin D1, early growth response 1 , and the polypeptide $\mathrm{N}$-acetylgalactosaminyltransferase 3, via the MEK/ERK signaling pathway [21-26]. Furthermore, recent studies indicate that the phosphate transporter Pit2 modulates FGF23 secretion in bone in response to high extracellular phosphate $[27,28]$. This action seems to be mediated by a signaling pathway other than MEK/ERK [20]. The kidney is also responsive to alterations in extracellular phosphate [19]. Other studies suggest that FGFR and Akt/mTOR signaling may be also involved in extracellular phosphate sensing $[24,29]$. Here, we wanted to investigate if extracellular phosphate altered the effect of systemic and constitutive activation of Jak1/Stat3 signaling on phosphate metabolism.

\section{Materials and Methods}

Mice Origin and Handling

The $\mathrm{C} 3 \mathrm{HeB} / \mathrm{FeJ}-\mathrm{Jakl}^{\mathrm{S} 645 \mathrm{PMhda}}\left(\mathrm{Jakl}^{\mathrm{S} 645 \mathrm{P}+\text { - }}\right)$ mouse line was generated within the Munich ENU Mutagenesis Project and backcrossed to the $\mathrm{C} 3 \mathrm{HeB} / \mathrm{FeJ}$ genetic background for at least 10 generations [30]. For this study, Jak1 $1^{\mathrm{S} 645 \mathrm{P}+/-}$ males and wild-type (WT) females were mated. At 4-6 weeks of age, the mice were put on a customized standard phosphate diet $(0.6 \%$ phosphorous, $1 \%$ calcium, and $600 \mathrm{IU} / \mathrm{kg}$ cholecalciferol; ssniff Spezialdiäten $\mathrm{GmbH}$, Germany) until harvesting around 12-14 weeks of age. All the experiments were performed before the animals developed the autoimmune phenotype described previously [30]. For the phosphate challenge and urine collection, at 12-13 weeks, mice were placed in metabolic cages (Tecniplast Inc., Italy). After 24-h adaptation, mice were put on either a standard phosphate diet (0.6\% phosphorous), a low phosphate diet ( $0.1 \%$ phosphorous), or a high phosphate diet (1.2\% phosphorous; all with $1 \%$ calcium and $600 \mathrm{IU} / \mathrm{kg}$ cholecalciferol, provided by ssniff Spezialdiäten $\mathrm{GmbH}$, Germany). In these diets, the source of phosphorous is of inorganic origin. After $48 \mathrm{~h}$, urine was collected during $24 \mathrm{~h}$. The phosphate diet of organic origin was obtained from Kliba Nafag (Cat. No.: 3433, 


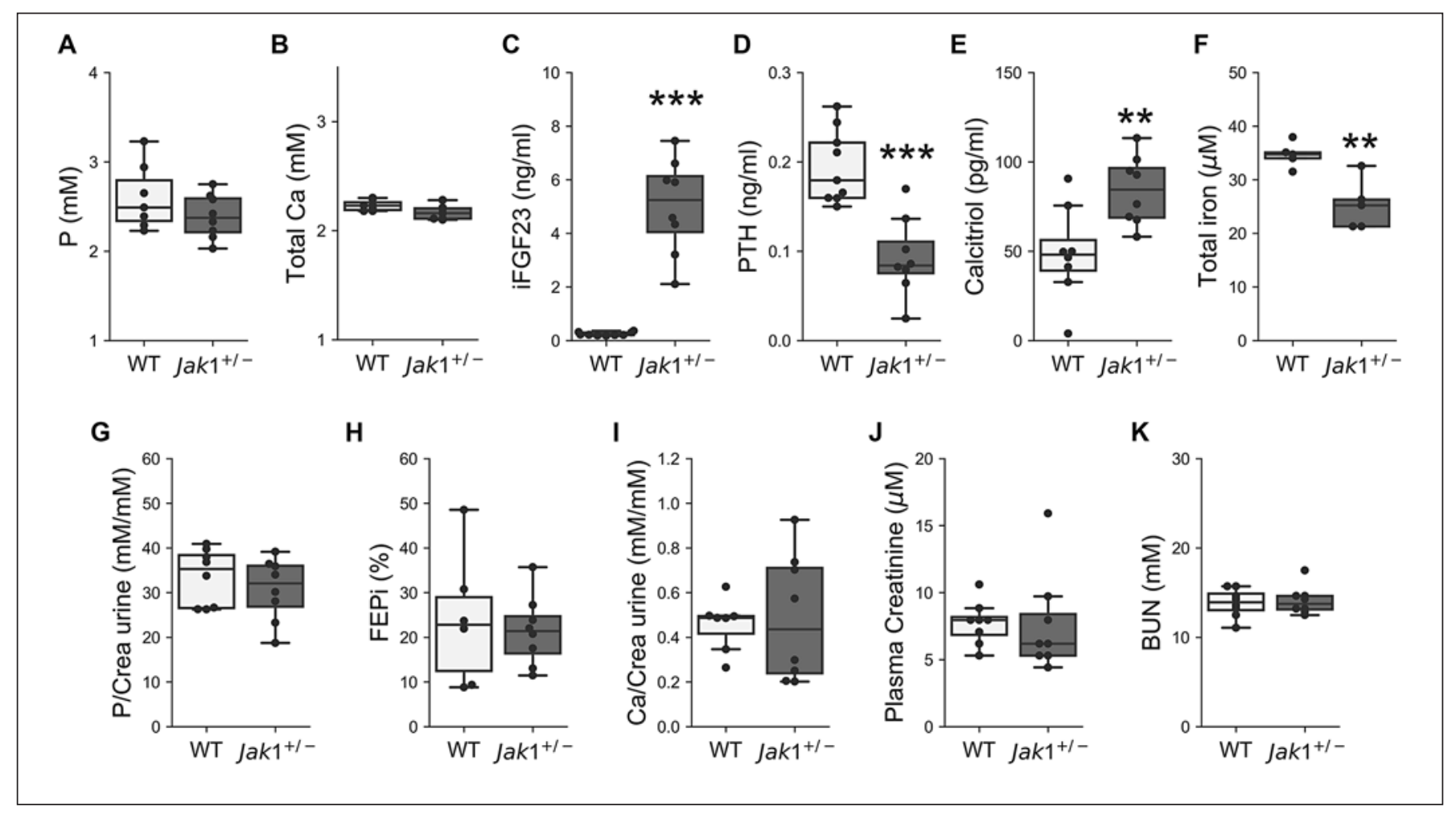

Fig. 1. Alteration in mineral metabolism parameters following Jak1/Stat3 activation in female mice under a standard phosphate

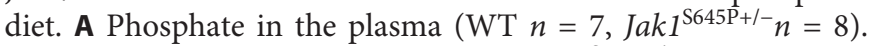
B Total calcium in the plasma (WT $\left.n=7, \mathrm{Jakl}^{\mathrm{S} 645 \mathrm{P}+/-} n=7\right)$. C, D Intact FGF23 and PTH in the plasma (WT $n=9, \operatorname{Jakl}^{\mathrm{S} 645 \mathrm{P}+/-} n=8$ ). E Calcitriol in the plasma (WT $n=8, \operatorname{Jakl}^{\mathrm{S645 \textrm {P } + l -}} n=8$ ). F Total iron in the plasma (WT $n=5, J a k 1^{\mathrm{S}_{645 \mathrm{P}+/-}} n=5$ ). G-I Phosphate/creati-

Granovit AG, Switzerland; $0.8 \%$ phosphorous, $1.05 \%$ calcium, and $800 \mathrm{IU} / \mathrm{kg}$ cholecalciferol). For blood and organ collection, mice were anesthetized with $2 \%$ isoflurane prior to collection of blood and killed by exsanguination under $4 \%$ isoflurane for collection or organs. Organs that were not directly used for experiments were rapidly snap-frozen and stored at $-80^{\circ} \mathrm{C}$ until analysis. The blood was centrifuged at $4^{\circ} \mathrm{C}$ for collection of plasma, aliquoted, and rapidly stored at $-80^{\circ} \mathrm{C}$ until analysis.

\section{Blood and Urine Parameters}

Enzymatic creatinine in the plasma, creatinine in 24-h urine, calcium in 24-h urine and plasma, phosphorous in 24-h urine and plasma, and blood urea nitrogen (BUN) and total iron were measured in the Zurich Integrative Rodent Physiology platform of the University of Zurich using the UniCel DxC 800 Synchron System (Beckman Coulter, Brea, CA, USA). Intact FGF23 and PTH levels in plasma were quantified by ELISA using the Mouse/Rat FGF-23 (Intact) Kit (Cat. No.: 60-6800, Immutopics International, San Clemente, CA, USA) and the mouse intact PTH ELISA kit (Cat. No.: 60-2305, Immutopics International, San Clemente, CA, USA), respectively. Calcitriol levels in plasma were determined by radioimmunoassay with the 1,25-Dihydroxy Vitamin D RIA kit (Cat. No.: AA-54F1, Immunodiagnostic System, Frankfurt, Germany). nine and calcium/creatinine in the urine and fractional excretion of phosphate (WT $\left.n=8, \operatorname{Jakl}^{\mathrm{S} 645 \mathrm{P}+/-} n=8\right)$. J, K Plasma creatinine and BUN (WT $n=8, J a k 1^{\mathrm{S} 645 \mathrm{P}+1-} n=8$ ). WT littermates, Jak1 ${ }^{+/-}$: $J a k 1^{\mathrm{S} 645 \mathrm{P}+/-}$ mice. Differences due to genotype: ${ }^{* *} p$ value $<0.01$ and $* * * p$ value $<0.001$. FGF23, fibroblast growth factor 23 ; PTH, parathyroid hormone; BUN, blood urea nitrogen; WT, wild-type.

\section{Statistical Analysis}

All experiments contained littermates with controls and experimental groups randomly assigned. Statistical significance was calculated by a 2-tailed Student's $t$-test or 2-way ANOVA using a post hoc test (Tukey HSD) for multiple comparisons. Statistical outliers were identified as greater or lower than the mean \pm 3 times the interquartile range and discarded from analysis. All statistical analyses were performed using Python programming language 3.7.3 (Spyder, Anaconda, USA) and R statistical language 4.0.2 (R Studio Inc, Boston, MA, USA). The number of biological replicates is described in the figure legends.

\section{Results}

\section{Mineral Metabolism in Female Mice following Jak1/ \\ Stat3 Activation}

Recently, we showed that the Jak1/Stat3 signaling pathway is involved in mineral metabolism, and constitutive and systemic activation of this pathway leads to very high intact FGF23 levels, low PTH, and high calcitriol lev- 


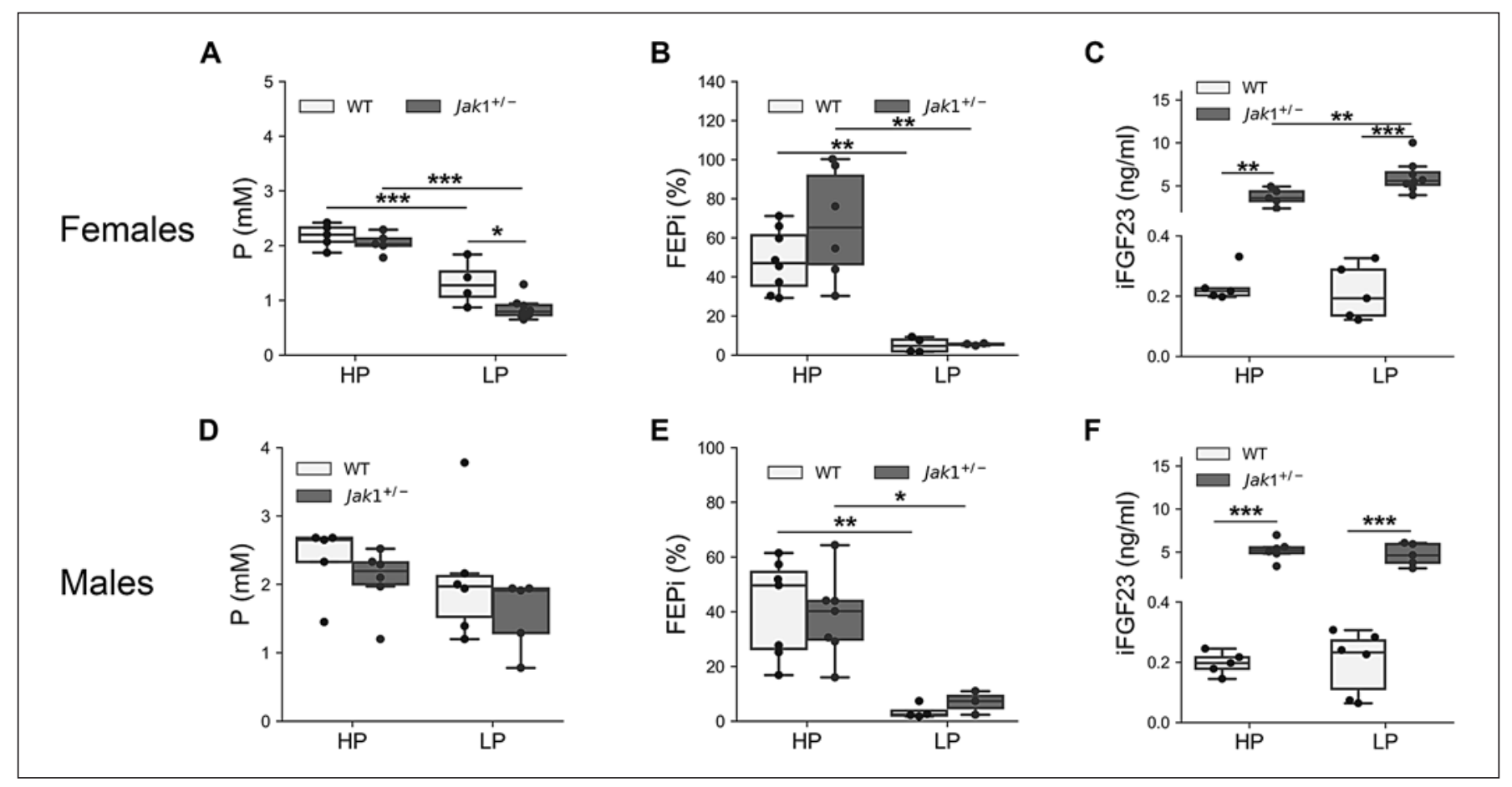

Fig. 2. Impact on phosphate and FGF23 of a dietary phosphate challenge with a low and a high phosphate diet during 2 days following Jak1/Stat3 activation. A Phosphate in the plasma in female mice (WT HP $n=5, J a k 1^{\mathrm{S} 645 \mathrm{P}+/-} \mathrm{HP} n=5$, WT LP $n=4, J a k 1^{\mathrm{S} 645 \mathrm{P}+/-}$ LP $n=8$ ). B Fractional excretion of phosphate in female mice (WT HP $n=8, J a k 1^{\mathrm{S} 645 \mathrm{P}+1-} \mathrm{HP} n=8$, WT LP $n=4, \mathrm{Jakl}^{\mathrm{S} 645 \mathrm{P}+1-} \mathrm{LP} n=$ 3 ). C Intact FGF23 in the plasma in female mice (WT HP $n=5$, $J a k 1^{\mathrm{S} 645 \mathrm{P}+1-} \mathrm{HP} n=6$, WT LP $\left.n=6, \mathrm{Jak}^{\mathrm{S} 645 \mathrm{P}+1-} \mathrm{LP} n=5\right)$. D Phosphate in the plasma in male mice (WT HP $n=5, J a k 1^{\mathrm{S} 645 \mathrm{P}+/-} \mathrm{HP}$

els in male mice [6]. Here, we investigated the impact of Jak1/Stat3 activation on mineral metabolism in female mice. As in males, no differences in phosphate and calcium in the plasma were observed between genotypes (Fig. 1A, B). Jak1/Stat3 activation led to very high intact FGF23 levels in the plasma and lower PTH and total iron levels and higher calcitriol levels in female Jak1 $1^{\mathrm{S} 645 \mathrm{P}+/-}$ mice (Fig. 1C-F). Despite the very high intact FGF23 levels, female Jak $1^{\mathrm{S} 645 \mathrm{P}+/-}$ mice showed normophosphaturia and a similar fractional excretion of phosphate to WT mice (Fig. 1G, H). Female Jak1 ${ }^{\mathrm{S} 645 \mathrm{P}+/-}$ mice showed normocalciuria, and the two renal function markers were investigated; creatinine in the plasma and BUN showed no alterations (Fig. 1I-K). Therefore, female $\mathrm{Jakl}^{\mathrm{S} 645 \mathrm{P}+1-}$ mice showed a similar impact on mineral metabolism parameters following systemic Jak1 activation than male mutant mice. Next, we focused on WT animals to assess if there were significant differences in phosphate metabolism parameters depending on sex at baseline conditions, $n=6$, WT LP $\left.n=6, \operatorname{Jakl}^{\mathrm{S} 645 \mathrm{P}+/-} \mathrm{LP} n=5\right)$. E Fractional excretion of phosphate in male mice (WT HP $n=7, J_{a k 1}{ }^{\mathrm{S} 645 \mathrm{P}+1-} \mathrm{HP} n=8$, WT LP $n=4$, Jak1 $1^{\mathrm{S} 645 \mathrm{P}+/-}$ LP $\left.n=3\right)$. F Intact FGF23 in plasma in male mice (WT HP $n=5$, Jak $1^{\mathrm{S} 645 \mathrm{P}+/-} \mathrm{HP} n=6$, WT LP $n=6$, $\left.J a k 1^{\mathrm{S} 645 \mathrm{P}+/-} \mathrm{LP} n=5\right)$. WT littermates, Jak1 $1^{+/-}: \mathrm{Jak}_{1}{ }^{\mathrm{S} 645 \mathrm{P}+1-}$ mice. HP: high phosphate diet $(1.2 \% \mathrm{w} / \mathrm{w})$, LP: low phosphate diet $(0.1 \%$ w/w). Two-way ANOVA analysis followed by Tukey HSD test: ${ }^{*} p$ value $<0.05,{ }^{* *} p$ value $<0.01$, and ${ }^{* * *} p$ value $<0.001$. FGF23, fibroblast growth factor 23; WT, wild-type.

comparing these data with the previously published [6]. No differences were observed in phosphate, total calcium, intact FGF23, and calcitriol levels in plasma and urinary phosphate and calcium excretion (online suppl. Table 1; see www.karger.com/doi/10.1159/000518488 for all online suppl. material). Yet, male showed significantly higher PTH levels in plasma than female mice. Total iron and creatinine in plasma were similar between sex, whereas BUN was lower in female mice.

\section{Phosphate Feeding Challenge}

To further understand the role of the Jak1/Stat 3 signaling pathway in mineral homeostasis, we examined if a dietary phosphate challenge altered mineral metabolism parameters differently during constitutive Jak1/ Stat 3 activation. Therefore, we tested the impact of feeding a high $(1.2 \% \mathrm{w} / \mathrm{w})$ and low $(0.1 \% \mathrm{w} / \mathrm{w})$ phosphate diet for $48 \mathrm{~h}$ in $J a k 1^{\mathrm{S} 645 \mathrm{P}+/-}$ mice compared to WT mice for both genders. Both genotypes showed significantly lower 


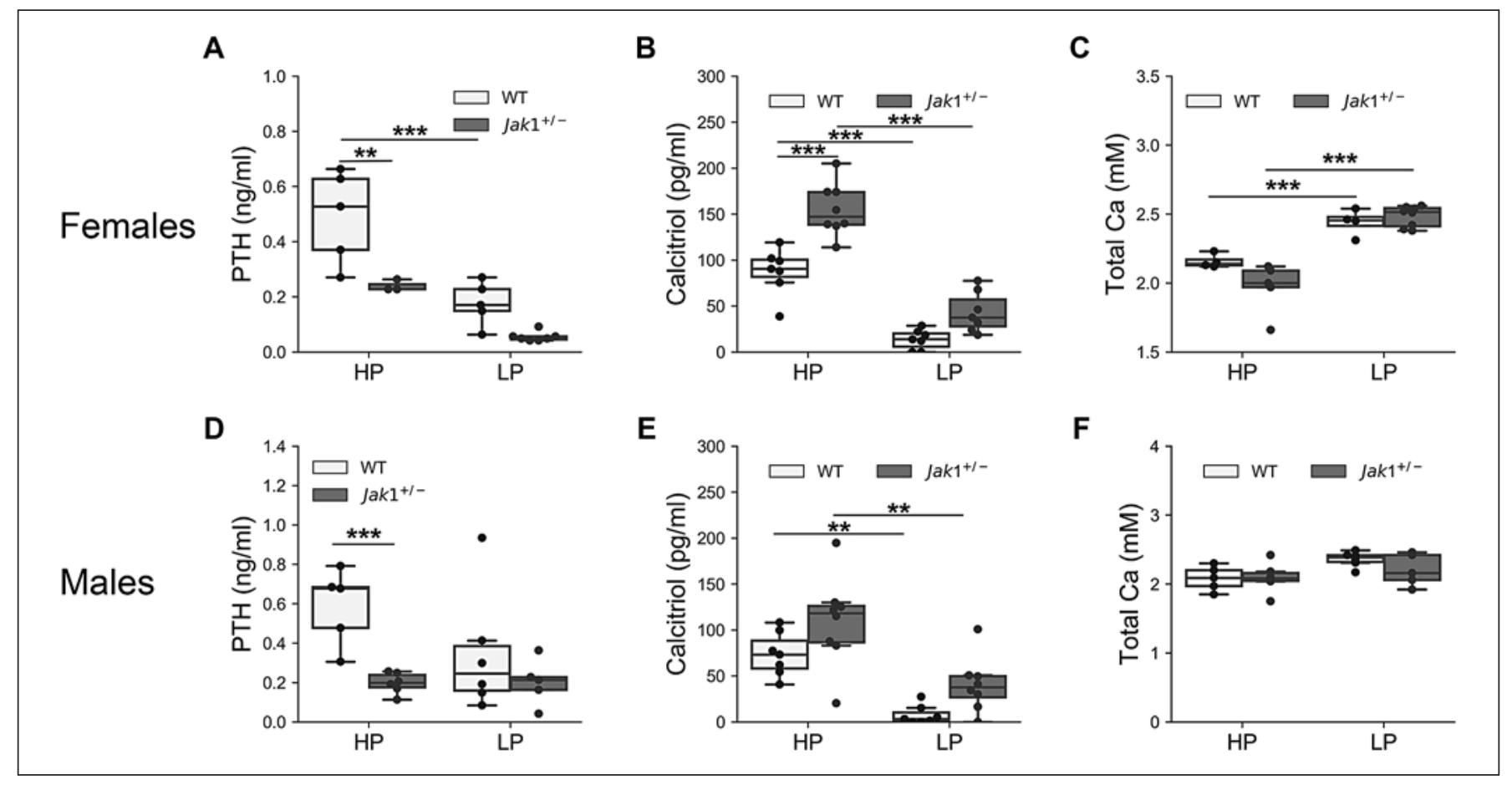

Fig. 3. Impact on PTH, calcitriol and calcium of a dietary phosphate challenge with a low and a high phosphate diet during 2 days following Jak1/Stat 3 activation. A PTH in the plasma in female mice (WT HP $n=5, J a k 1^{\mathrm{S} 645 \mathrm{P}+/-} \mathrm{HP} n=6$, WT LP $n=6$, Jak $1^{\mathrm{S} 645 \mathrm{P}+1-}$ LP $n=5$ ). B Calcitriol in the plasma in female mice (WT HP $n=7$, $J a k 1^{\text {S645P+l- }}$ HP $n=8$, WT LP $n=7, J a k 1^{\text {S645P+l- }}$ LP $\left.n=7\right)$. C Total calcium in the plasma in female mice (WT HP $n=5, J a k 1^{\mathrm{S} 645 \mathrm{P}+1-}$ HP $n=5$, WT LP $\left.n=4, J a k 1^{\mathrm{S} 645 \mathrm{P}+/-} \operatorname{LP} n=8\right)$. D PTH in the plasma in male mice (WT HP $n=5, J a k 1^{\mathrm{S} 645 \mathrm{P}+/-} \mathrm{HP} n=6$, WT LP $n=6$,

phosphate levels in plasma under a low phosphate diet compared to a high phosphate diet for female mice, and same tendency was observed in male mice (Fig. 2A, D). When the animals were challenged with a low phosphate diet, Jak1/Stat3 activation led to lower phosphate levels in blood than in WT littermates only in female mice (Fig. 2A). Fractional phosphate excretion was only dependent on the diet consumed, being higher under a high than a low phosphate diet for both genders and genotypes (Fig. 2B, E). Intact FGF23 remained higher in $J a k 1^{\mathrm{S} 645 \mathrm{P}+1-}$ mice under both a low and a high phosphate diet for both genders, and even increased in female $J a k 1^{\mathrm{S} 645 \mathrm{P}+/-}$ mice under a low phosphate diet when compared to levels in female mice consuming a high phosphate diet (Fig. 2C, F). PTH was lower in Jak1 $1^{\mathrm{S} 645 \mathrm{P}+/-}$ mice than WT under a high phosphate diet for both genders, but no significant differences could be detected between genotypes in animals fed a low phosphate diet (Fig. 3A, D). Calcitriol was higher in animals fed a high
$\left.J a k 1^{\mathrm{S} 645 \mathrm{P}+/-} \mathrm{LP} n=5\right)$. E Calcitriol in the plasma in male mice (WT HP $n=7, J a k 1^{\text {S645P+l- }}$ HP $n=8$, WT LP $n=5$, Jak $1^{\text {S645P+l- }}$ LP $n=$ 8). F Total calcium in the plasma in male mice (WT HP $n=5$, $J a k 1^{\mathrm{S} 645 \mathrm{P}+/-} \mathrm{HP} n=6$, WT LP $n=6$, Jak1 $\left.1^{\mathrm{S} 645 \mathrm{P}+/-} \mathrm{LP} n=5\right)$. WT littermates, Jak1 $1^{+/-}$: Jak1 ${ }^{\mathrm{S} 645 \mathrm{P}+/-}$ mice. HP: high phosphate diet $(1.2 \%$ $\mathrm{w} / \mathrm{w}), \mathrm{LP}$ : low phosphate $\operatorname{diet}(0.1 \% \mathrm{w} / \mathrm{w})$. Two-way ANOVA analysis followed by Tukey HSD test: ${ }^{* *} p$ value $<0.01$ and ${ }^{* * *} p$ value $<0.001$. PTH, parathyroid hormone; WT, wild-type.

phosphate diet than in animals fed a low phosphate diet for both genotypes and both genders (Fig. 3B, E). This is probably due, at least in female mice, to a lower availability of calcium under a high phosphate diet than under a low phosphate diet (Fig. 3C, F) and triggered by PTH modulating calcitriol metabolism. Under a high phosphate diet, calcitriol levels were higher in female Jak $1^{\mathrm{S} 645 \mathrm{P}+1-}$ mice than WT littermates, whereas a low phosphate diet only provoked a tendency to higher calcitriol levels during Jak1/Stat 3 activation $(p=0.0971)$. No differences due to genotype were observed in male mice. Last, we focused on WT animals to assess if there were significant differences in phosphate metabolism parameters depending on sex in mice challenged to different phosphate content in the diet. Phosphate, total calcium, iFGF23, PTH, and calcitriol in plasma as well as fractional phosphate excretion reacted similarly for both genders, when challenged with a low and a high phosphate diet. 


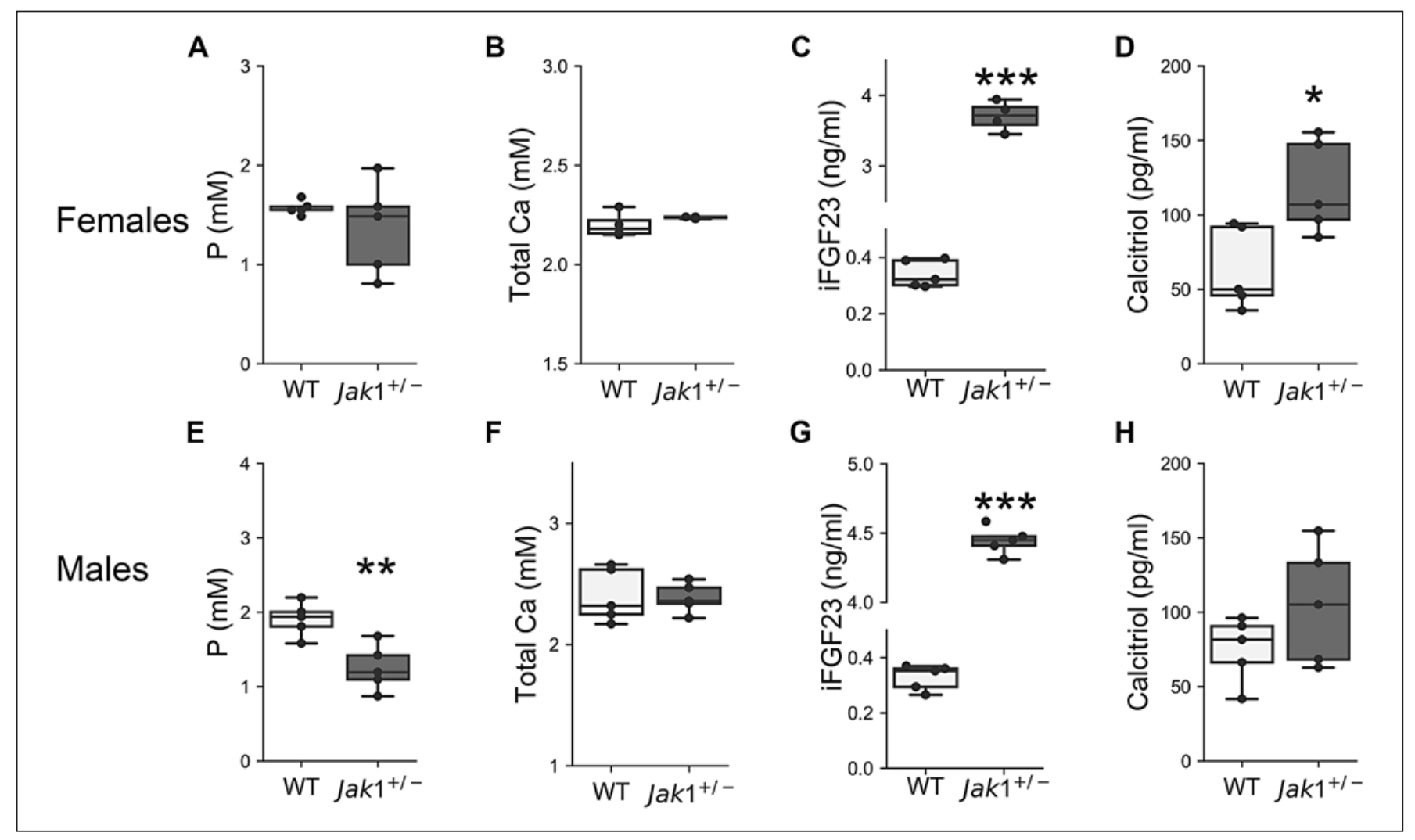

Fig. 4. Alteration in mineral metabolism parameters following Jak1/Stat 3 activation in mice under a standard phosphate diet of organic source. A-D Phosphate, total calcium, intact FGF23, and calcitriol in the plasma in female mice (WT $n=5, \operatorname{Jakl}^{\mathrm{S} 645 \mathrm{P}+/-} n=5$ ). E-H Phosphate, calcium, intact FGF23, and calcitriol in the plasma in male mice (WT $n=5$, Jak1 ${ }^{\mathrm{S} 645 \mathrm{P}+1-} n=5$ ). WT littermates, Jak1+/- $: J a k 1^{\mathrm{S} 645 \mathrm{P}+/-}$ mice. Differences due to the genotype: ${ }^{*} p$ value $<0.05,{ }^{* *} p$ value $<0.01$, and ${ }^{* * *} p$ value $<0.001$. FGF23, fibroblast growth factor 23 ; PTH, parathyroid hormone; WT, wild-type.

\section{Impact of Phosphate Bioavailability on Mineral}

\section{Metabolism following Jak1 Activation}

We next showed that feeding animals with a phosphorous diet of lower bioavailability (organic vs. inorganic origin) did not change much phosphate metabolism alterations observed following systemic Jak1/Stat3 activation. Under a standard phosphate diet of organic origin, female $J a k 1^{\mathrm{S} 645 \mathrm{P}+1-}$ mice were normophosphatemic and normocalcemic (Fig. 4A, B), whereas male Jak1 ${ }^{\mathrm{S} 645 \mathrm{P}+/-}$ mice were normocalcemic, but showed hypophosphatemia (Fig. 4E, F). Intact FGF23 showed very high levels in plasma following Jak1/Stat3 activation for both genders (Fig. 4C, G). Female Jak1 ${ }^{\mathrm{S} 645 \mathrm{P}+/-}$ mice showed higher calcitriol levels than their WT littermates (Fig. 4D), and male $J a k 1^{5645 \mathrm{P}+/-}$ showed a tendency to higher calcitriol levels, which did not reach statistical significance (Fig. 4H). Next, we focused on WT animals to assess if there were significant differences in phosphate metabolism param- eters depending on sex, as previously. No differences were observed in total calcium, intact FGF23 and calcitriol levels in plasma. Yet, male showed significantly higher phosphate levels in plasma than female mice $(p<0.05)$.

\section{Discussion/Conclusion}

Few studies have been focusing on the role of sex and gender in phosphate metabolism. Some studies report similar phosphate levels in plasma in women and men [16], whereas other report women having slightly higher phosphate levels [31]. Other found higher phosphate levels in women aged over 45 years than men $[32,33]$. Of note, major differences exist how blood samples were collected (e.g., fasted vs. nonfasted, timed collection vs. random) that likely impact outcomes as phosphate levels, as well as the levels of hormones regulating phosphate bal- 
Fig. 5. Scheme of possible mechanisms linking Jak1/Stat3 activation and phosphate homeostasis. Constitutive activation of Jak1/Stat3 signaling leads to low PTH, low total iron levels, and high calcitriol production. Local inflammation is detected in the liver and bone. The liver secretes high levels of intact FGF23 and excessive levels of the C-terminal FGF23 fragment. The latter probably inhibits the physiological action of FGF23 on the kidney, resulting in similar phosphate reabsorption in mutant and WT animals. Secretion of FGF23 in the bone is unchanged. Low iron might contribute to high C-terminal FGF23 fragment levels. PTH, parathyroid hormone; FGF23, fibroblast growth factor 23.

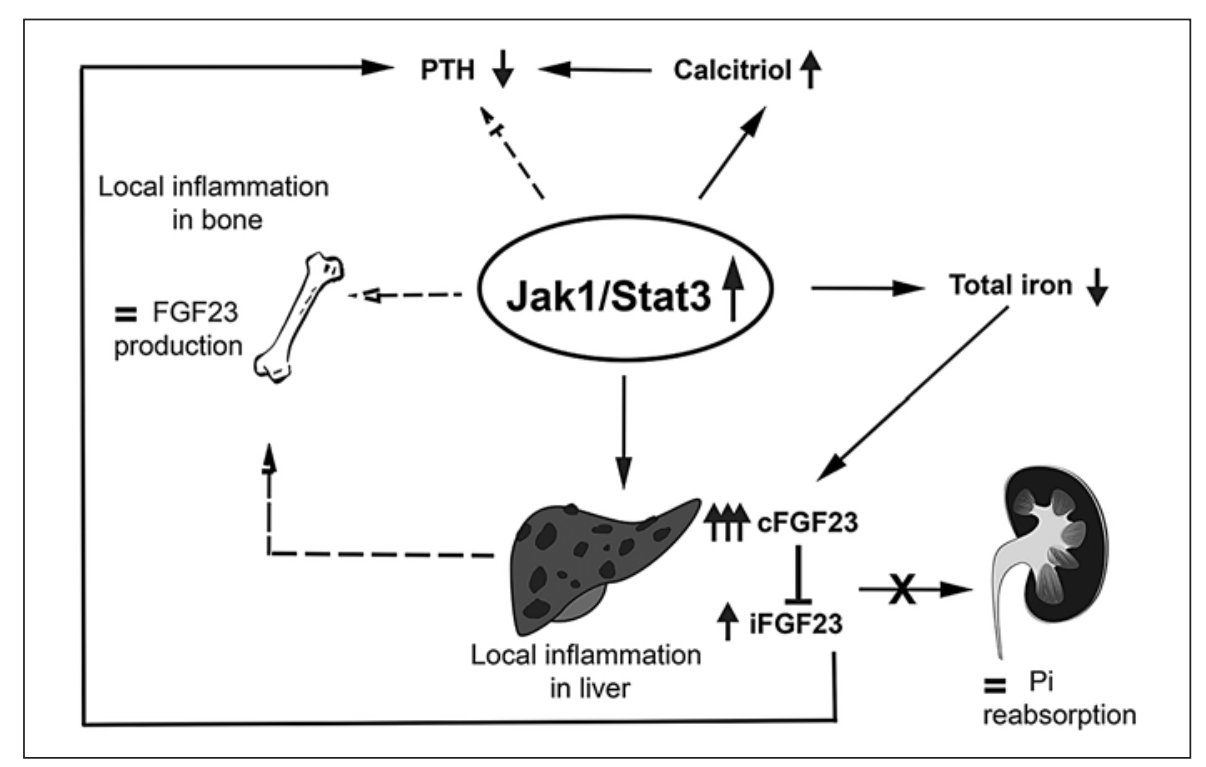

ance show circadian rhythms [34]. The sex biological aspects dependence on phosphate handling have not been studied in detail [35]. Estrogen is known to have several actions on phosphate metabolism: it increases intestinal phosphate absorption and has a phosphaturic action in the kidney probably mediated by FGF $23[18,36,37]$. A recent study in mice shows that aged female mice increased their intact FGF23 levels in plasma after an 8 weeks phosphate challenge when compared to younger mice without changing their phosphate plasma levels, whereas male mice did not show this response [38]. The authors found similar phosphate and intact FGF23 levels in plasma due to sex for both age studies, at 16 and 78 weeks of age. Therefore, it seems that FGF23 production after a phosphate challenge may be sex dependent in older ages. Here, while investigating WT mice aged 12-14 weeks, we also found similar phosphate and intact FGF23 levels between males and females when fed a phosphate diet of inorganic origin. Male mice fed a phosphate diet of organic origin showed higher levels in the plasma than those in female mice. In addition, we found that male and female mice have similar urinary phosphate excretion and calcium and calcitriol levels in the plasma. The only difference was observed in PTH, with male animals having higher levels than female animals. To our best knowledge, this sex difference in PTH levels has not been found in humans.

We further investigated if the modulation of phosphate handling following Jak1 activation was different in females when compared to the effects reported in male mice. Previously, as summarized in Figure 5, we have shown in male mice that FGF23 production in the bone was not altered following Jak1/Stat 3 activation, but the liver produced very high FGF23 levels, triggered by the inflammation detectable in this organ [6]. Yet, the liver produced both higher C-terminal FGF23 fragments and high intact (active) FGF23 hormone, which resulted in no effect on phosphate excretion in the kidney, likely because of the inhibitory action of the C-terminal FGF23 fragment on active FGF23 signaling [10]. A 20-fold higher secretion of C-terminal FGF23 fragment versus active FGF23 blocked the phosphaturic action of FGF23. Jak1/Stat3 activation resulted also in low PTH levels and high calcitriol levels in the plasma. Here, female mice showed also very high intact FGF23 levels in the plasma, low PTH, and high calcitriol levels with no effect on phosphate and total calcium in the plasma. Despite the high intact FGF23 levels, urinary phosphate fractional excretion and the ratio of urinary phosphate to creatinine and calcium to creatinine were similar between genotypes, as summarized in Figure 5. We assume that this effect is due to a high C-terminal FGF23 production from the liver of female $J a k 1^{\mathrm{S} 645 \mathrm{P}+/-}$ mice as reported for male Jak $1^{\mathrm{S} 645 \mathrm{P}+/-}$ mice, which blocks the phosphaturic action of intact FGF23. Kidney function is maintained after Jak1/Stat 3 activation. Jak/Stat signaling plays a major role in erythropoiesis [8]; therefore, it was not surprising to find previously that $J a k 1^{\mathrm{S} 645 \mathrm{P}+/-}$ mice have slightly higher erythrocyte counts and lower hemoglobin levels for both male and female mice [30]. Additionally, total iron levels are decreased in both male and female mice, as reported here and previously [6]. Low iron has been shown to increase C-terminal 
fragment levels in the plasma [39]. Whether low iron contributes to the excessive levels of C-terminal FGF23 fragment after Jak/Stat activation remains to be established.

Systemic activation of the Jak1/Stat3 signaling pathway induces hypo- or normophosphatemia depending on the bioavailability of phosphate. Feeding a standard phosphate diet with high bioavailability (inorganic source) causes normophosphatemia [6], whereas feeding a diet with low bioavailability (organic source) provokes hypophosphatemia after Jak1/Stat3 activation [30]. Here, we also showed that low phosphate content in the diet provokes hypophosphatemia after Jak1/Stat 3 activation, but only in female mice. Moreover, intake of a diet with lower bioavailability (organic source) induced hypophosphatemia only in male mice. We hypothesize that the higher interindividual variability masks the finding that following Jak1/Stat3 activation, mice develop hypophosphatemia when they consume a phosphate diet with low bioavailability. Furthermore, in mutant mice, female and male, intact FGF23 levels remained high under both a low and a high phosphate diet. In contrast, calcitriol was only significantly higher, and PTH was lower, when $J a k 1^{\mathrm{S} 645 \mathrm{P} /{ }_{+}}$mice were fed a high phosphate diet, while these differences became smaller and nonsignificant on a low phosphate diet. These differences were again more marked in those groups of animals showing less interindividual variability. Therefore, although Jak1/Stat3 signaling modulates mineral homeostasis, it seems not to affect phosphate-sensitivity of PTH and calcitriol secretion. These hormones are primarily regulated by calcium levels in blood. However, intact FGF23 levels did not decrease in mutant mice despite overt hypophosphatemia, suggesting that intact FGF23 levels have become uncoupled from plasma phosphate levels and are triggered by another stimulus, probably inflammation as suggested previously [6].

In conclusion, here, we show that for both sexes, Jak1/ Stat3 activation leads to very high FGF23, low PTH, and high calcitriol levels in the plasma. Moreover, changes in extracellular phosphate did not change this response for both male and female mice. This study contributes to a better understanding of the role of Jak/Stat signaling in mineral metabolism and therefore to the understanding of phosphate metabolism.

\section{Acknowledgement}

Prof. Hrabě de Angelis (Helmholtz Zentrum München, Germany) is gratefully acknowledged for providing the $J a k 1^{\mathrm{S} 645 \mathrm{P}+/-}$ (C3HeB/FeJ-Jak1S645PMhda) mouse line, which was generated and funded by the following grants held by Prof. Hrabě de Angelis: German Federal Ministry of Education and Research (Infrafron- tier Grant 01KX1012, OSTEOPATH Grant 01EC1006B) and the German Center for Diabetes Research (DZD). We kindly thank Udo Schnitzbauer for technical support at the University of Zurich (Switzerland). The Zurich Integrative Rodent Physiology core facility for Rodent Phenotyping and the Histology Laboratory at the University of Zurich are also acknowledged.

\section{Statement of Ethics}

All procedures applied through this study were conducted according to the Swiss animal welfare laws and guidelines for animal care and approved by the Zurich Veterinary Office (Kantonales Veterinäramt) under the reference numbers ZH05/2013 and ZH156/2016.

\section{Conflict of Interest Statement}

The authors have no conflicts of interest to declare. CAW reports honoraria and grants from Bayer, Medice, Kyowa Kirin, and Chugai for projects unrelated to this study.

\section{Funding Sources}

The study was in part supported by the Swiss National Center of Competence in Research NCCR Kidney. C.H. was funded by the Swiss National Science Foundation and a grant from the Swiss National Science Foundation (176125) to C.A.W.

\section{Author Contributions}

N. Gehring and C. Bettoni conducted the experiments. C.A. Wagner designed the experiments, interpreted the results, and provided funding. I. Rubio-Aliaga designed the experiments, conducted the experiments, analyzed the data, interpreted the results, and wrote the manuscript. All the authors read the manuscript, contributed to editing, and approved the final version.

\section{Data Availability Statement}

The data that support the findings of this study are available from the corresponding author upon reasonable request.

\section{References}

Kidney Blood Press Res 2021;46:714-722 DOI: $10.1159 / 000518488$
1 Hu MC, Shiizaki K, Kuro-o M, Moe OW. Fibroblast growth factor 23 and Klotho: physiology and pathophysiology of an endocrine network of mineral metabolism. Annu Rev Physiol. 2013;75:503-33.

2 Bar L, Stournaras C, Lang F, Foller M. Regulahealth and disease. FEBS Lett. 2019 Aug; 593(15):1879-900.

3 Levi M, Gratton E, Forster IC, Hernando N, Wagner CA, Biber J, et al. Mechanisms of phosphate transport. Nat Rev Nephrol. 2019 Aug;15(8):482-500. tion of fibroblast growth factor 23 (FGF23) in
Jak1 Role in Mineral Metabolism Is Independent of Phosphate and Sex 
4 Vervloet M. Renal and extrarenal effects of fibroblast growth factor 23. Nat Rev Nephrol. 2019 Feb;15(2):109-20.

5 Berndt T, Kumar R. Phosphatonins and the regulation of phosphate homeostasis. Annu Rev Physiol. 2007;69:341-59.

6 Daryadel A, Ruiz PA, Gehring N, Stojanovic D, Ugrica M, Bettoni C, et al. Systemic Jak1 activation provokes hepatic inflammation and imbalanced FGF23 production and cleavage. FASEB J. 2021 Feb;35(2):e21302.

7 Fuente R, Gehring N, Bettoni C, Gil-Peña H, Alonso-Durán L, Michalke B, et al. Systemic Jak1 activation causes extrarenal calcitriol production and skeletal alterations provoking stunted growth. FASEB J. 2021;35(7):e21721.

8 O'Shea JJ, Schwartz DM, Villarino AV, Gadina M, McInnes IB, Laurence A. The JAKSTAT pathway: impact on human disease and therapeutic intervention. Annu Rev Med. 2015;66:311-28.

9 Umbach AT, Zhang B, Daniel C, Fajol A, Velic A, Hosseinzadeh Z, et al. Janus kinase 3 regulates renal 25-hydroxyvitamin D 1a-hydroxylase expression, calcitriol formation, and phosphate metabolism. Kidney Int. 2015 Apr;87(4):728-37.

10 Goetz R, Nakada Y, Hu MC, Kurosu H, Wang L, Nakatani T, et al. Isolated C-terminal tail of FGF23 alleviates hypophosphatemia by inhibiting FGF23-FGFR-Klotho complex formation. Proc Natl Acad Sci U S A. 2010 Jan 5; 107(1):407-12.

11 Mauvais-Jarvis F, Bairey Merz N, Barnes PJ, Brinton RD, Carrero JJ, DeMeo DL, et al. Sex and gender: modifiers of health, disease, and medicine. Lancet. 2020 Aug 22;396(10250): $565-82$.

12 GBD 2016 Causes of Death Collaborators. Global, regional, and national age-sex specific mortality for 264 causes of death, 1980-2016: a systematic analysis for the Global Burden of Disease Study 2016. Lancet. 2017 Sep 16; 390(10100):1151-210.

13 Heron M. Deaths: leading causes for 2017. Natl Vital Stat Rep. 2019 Jun;68(6):1-77.

14 Sederholm Lawesson S, Alfredsson J, Szummer K, Fredrikson M, Swahn E. Prevalence and prognostic impact of chronic kidney disease in STEMI from a gender perspective: data from the SWEDEHEART register, a large Swedish prospective cohort. BMJ Open. 2015 Jun 23;5(6):e008188.

15 Loh TP, Metz MP. Trends and physiology of common serum biochemistries in children aged 0-18 years. Pathology. 2015 Aug;47(5): 452-61.

16 Cirillo M, Ciacci C, De Santo NG. Age, renal tubular phosphate reabsorption, and serum phosphate levels in adults. N Engl J Med. 2008 Aug 21;359(8):864-6.
17 Webster R, Sheriff S, Faroqui R, Siddiqui F, Hawse JR, Amlal H. Klotho/fibroblast growth factor 23- and PTH-independent estrogen receptor- $\alpha$-mediated direct downregulation of NaPi-IIa by estrogen in the mouse kidney. Am J Physiol Renal Physiol. 2016 Aug 1; 311(2):F249-59.

$18 \mathrm{Xu} \mathrm{H}, \mathrm{Uno} \mathrm{JK}$, Inouye M, Xu L, Drees JB, Collins JF, et al. Regulation of intestinal NaPi-IIb cotransporter gene expression by estrogen. Am J Physiol Gastrointest Liver Physiol. 2003 Dec;285(6):G1317-24.

19 Michigami T, Kawai M, Yamazaki M, Ozono $\mathrm{K}$. Phosphate as a signaling molecule and its sensing mechanism. Physiol Rev. 2018 Oct 1; 98(4):2317-48.

20 Beck L, Beck-Cormier S. Extracellular phosphate sensing in mammals: what do we know? J Mol Endocrinol. 2020 Oct;65(3):R53-63.

21 Beck GR Jr, Zerler B, Moran E. Phosphate is a specific signal for induction of osteopontin gene expression. Proc Natl Acad Sci U S A. 2000 Jul 18;97(15):8352-7.

22 Beck GR Jr, Knecht N. Osteopontin regulation by inorganic phosphate is ERK1/2-, protein kinase C-, and proteasome-dependent. J Biol Chem. 2003 Oct 24;278(43):41921-9.

23 Kimata M, Michigami T, Tachikawa K, Okada T, Koshimizu T, Yamazaki M, et al. Signaling of extracellular inorganic phosphate upregulates cyclin D1 expression in proliferating chondrocytes via the $\mathrm{Na}+\mathrm{Pi}$ cotransporter Pit-1 and Raf/MEK/ERK pathway. Bone. 2010 Nov;47(5):938-47.

24 Yamazaki M, Ozono K, Okada T, Tachikawa K, Kondou H, Ohata Y, et al. Both FGF23 and extracellular phosphate activate Raf/MEK/ ERK pathway via FGF receptors in HEK293 cells. J Cell Biochem. 2010 Dec 1;111(5): 1210-21.

25 Nishino J, Yamazaki M, Kawai M, Tachikawa K, Yamamoto K, Miyagawa K, et al. Extracellular phosphate induces the expression of dentin matrix protein 1 through the FGF receptor in osteoblasts. J Cell Biochem. 2017 May;118(5):1151-63.

26 Takashi Y, Kosako H, Sawatsubashi S, Kinoshita Y, Ito N, Tsoumpra MK, et al. Activation of unliganded FGF receptor by extracellular phosphate potentiates proteolytic protection of FGF23 by its O-glycosylation. Proc Natl Acad Sci U S A. 2019 Jun 4;116(23): 11418-27.

27 Bon N, Couasnay G, Bourgine A, Sourice S, Beck-Cormier S, Guicheux J, et al. Phosphate (Pi)-regulated heterodimerization of the high-affinity sodium-dependent $\mathrm{Pi}$ transporters PiT1/Slc20a1 and PiT2/Slc20a2 underlies extracellular Pi sensing independently of Pi uptake. J Biol Chem. 2018 Feb 9;293(6): 2102-14.
28 Bon N, Frangi G, Sourice S, Guicheux J, BeckCormier S, Beck L. Phosphate-dependent FGF23 secretion is modulated by PiT2/Slc20a2. Mol Metab. 2018 May;11:197-204.

29 Kawai M, Kinoshita S, Ozono K, Michigami $\mathrm{T}$. Inorganic phosphate activates the AKT/ mTORC1 pathway and shortens the life span of an a-Klotho-deficient model. J Am Soc Nephrol. 2016 Sep;27(9):2810-24.

30 Sabrautzki S, Janas E, Lorenz-Depiereux B, Calzada-Wack J, Aguilar-Pimentel JA, Rathkolb B, et al. An ENU mutagenesis-derived mouse model with a dominant Jak1 mutation resembling phenotypes of systemic autoimmune disease. Am J Pathol. 2013 Aug;183(2): 352-68.

31 Onufrak SJ, Bellasi A, Cardarelli F, Vaccarino $\mathrm{V}$, Muntner P, Shaw LJ, et al. Investigation of gender heterogeneity in the associations of serum phosphorus with incident coronary artery disease and all-cause mortality. Am J Epidemiol. 2009 Jan 1;169(1):67-77.

32 Yoo KD, Kang S, Choi Y, Yang SH, Heo NJ, Chin HJ, et al. Sex, age, and the association of serum phosphorus with all-cause mortality in adults with normal kidney function. Am J Kidney Dis. 2016 Jan;67(1):79-88.

33 Koek WNH, Campos-Obando N, van der Eerden BCJ, de Rijke YB, Ikram MA, Uitterlinden AG, et al. Age-dependent sex differences in calcium and phosphate homeostasis. Endocr Connect. 2021 Mar;10(3):273-82.

34 Egli-Spichtig D, Wagner CA. The rhythm of healthy kidneys. Pflugers Arch. 2019 Sep; 471(9):1159-60.

35 Khalil R, Kim NR, Jardi F, Vanderschueren D, Claessens F, Decallonne B. Sex steroids and the kidney: role in renal calcium and phosphate handling. Mol Cell Endocrinol. 2018 Apr 15;465:61-72.

36 Faroqui S, Levi M, Soleimani M, Amlal H. Estrogen downregulates the proximal tubule type IIa sodium phosphate cotransporter causing phosphate wasting and hypophosphatemia. Kidney Int. 2008 May;73(10): 1141-50.

37 Carrillo-López N, Román-García P, Rodríguez-Rebollar A, Fernández-Martín JL, Naves-Díaz M, Cannata-Andía JB. Indirect regulation of $\mathrm{PTH}$ by estrogens may require FGF23. J Am Soc Nephrol. 2009 Sep;20(9): 2009-17.

38 Tippen SP, Noonan ML, Ni P, Metzger CE, Swallow EA, Sacks SA, et al. Age and sex effects on FGF23-mediated response to mild phosphate challenge. Bone. 2021 Feb 19;146: 115885.

39 Edmonston D, Wolf M. FGF23 at the crossroads of phosphate, iron economy and erythropoiesis. Nat Rev Nephrol. 2020 Jan; 16(1): 7-19. 